\title{
Impaired quality of life in treatment-seeking obese children of Dutch, Moroccan, Turkish and Surinamese descent
}

\author{
Nalini NE Radhakishun ${ }^{1,2, *}$, Maartie de Wit ${ }^{3}$, Mariska van Vliet ${ }^{4}$, Ines A von Rosenstiel ${ }^{1}$, \\ Jos $\mathrm{H}_{\text {Beijnen }}{ }^{5}$, Dees PM Brandjes ${ }^{6}$ and Michaela Diamant ${ }^{2}$ \\ 'Department of Pediatrics, Slotervaart Hospital, Louwesweg 6, 1066 EC Amsterdam, The Netherlands: ${ }^{2}$ Diabetes \\ Center/Department of Internal Medicine, VU University Medical Centre (VUMC), Amsterdam, The Netherlands: \\ ${ }^{3}$ Department of Medical Psychology, VU University, Amsterdam, The Netherlands: ${ }^{4}$ Department of Internal Medicine, \\ Leiden University Medical Center, Leiden, The Netherlands: ${ }^{5}$ Department of Pharmacy \& Pharmacology, Slotervaart \\ Hospital, Amsterdam, The Netherlands: ${ }^{6}$ Department of Internal Medicine, Slotervaart Hospital, Amsterdam, \\ The Netherlands
}

Submitted 1 July 2014: Final revision received 3 January 2015: Accepted 28 May 2015: First published online 24 July 2015

\begin{abstract}
Objective: To determine the health-related quality of life (HRQOL) of overweight and obese multi-ethnic children compared with normal-weight children; and to investigate differences in HRQOL between self- and parent-proxy reports and ethnic groups.

Design: Prospective cross-sectional study.

Setting: Out-patient clinic where children and their parents filled out a validated HRQOL questionnaire (KIDSCREEN-52) and height, weight, waist circumference and fat percentage were measured.

Subjects: Overweight and obese children, aged 8-18 years (mean BMI $Z$-score $3 \cdot 2$ (SD 0.6)), from the obesity out-patient clinic.

Results: Three hundred and eight self- and 213 parent-proxy reported questionnaires were completed. Global HRQOL and the Physical Wellbeing, Moods \& Emotions and Self-Perception subscales were markedly reduced in our multi-ethnic obese cohort, relative to the Dutch reference values. Parent proxies reported significantly lower on the global HRQOL and the Physical Wellbeing, Moods \& Emotions and Bullying subscales. In Caucasian children, multivariate analyses showed that BMI was associated with the quality-of-life subscales Moods \& Emotions, Self-Perception and Bullying.

Conclusions: HRQOL was markedly reduced in our multi-ethnic overweight and obese out-patient clinic cohort, with significantly lower parent-proxy scores compared with self-reported scores. We believe intervention programmes aiming to improve HRQOL should be directed to both parents and children, while ethnicspecific programmes to enhance HRQOL seem of less importance.
\end{abstract}

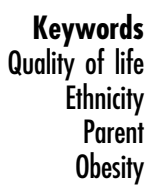

The obesity pandemic affects all age groups and imposes a large societal burden by conferring high risk for serious morbidities including diabetes, CVD and cancer ${ }^{(1)}$. In the Netherlands, almost one child out of seven is either overweight $(10 \%)$ or obese $(3 \%)^{(2)}$ and this figure is even higher in non-white children, mainly originating from Morocco, Turkey and Suriname ${ }^{(3,4)}$. The increasing prevalence and severity of obesity in children and adolescents has resulted in greater emphasis on the prevention and treatment of obesity and its accompanying cardiometabolic risk factors and associated co-morbidities (i.e. CVD, type 2 diabetes mellitus, sleep apnoea and renal impairment) ${ }^{(5)}$.
In addition to these physical health problems, obesity also poses a considerable risk of depression, psychological stress and poor developmental adaptation, which may contribute to (development of) other health problems ${ }^{(5,6)}$. One of the most prevalent and immediate consequences of obesity in childhood is its direct impact on health-related quality of life (HRQOL) ${ }^{(7-9)}$. HRQOL is a comprehensive, multidimensional construct encompassing physical and psychosocial well-being. Previous studies among clinical cohorts of morbidly obese children have shown that these children have a significantly lower HRQOL, as compared with lean children, that is lower than for children with cystic fibrosis, epilepsy and type 1 
diabetes, and even comparable to that of children who are treated for malignancies ${ }^{(10)}$.

In previous studies, BMI was inversely associated with HRQOL $^{(7-9)}$. However, with regard to ethnic-specific differences in HRQOL among obese children, little and contradictive evidence is available ${ }^{(8,10,11)}$. For example, one study found that Afro-American overweight adolescents had less impairments in quality of life (QOL) than overweight white adolescents ${ }^{(9)}$, while others reported no significant associations between weight classification and race/ethnicity for QOL in fifth-grade children ${ }^{(11)}$. One of the few European studies found that parents of 5-6-year-old children of Dutch origin reported lower scores on several HRQOL subscales, relative to non-Dutch children ${ }^{(12)}$. However, in the cited study, over $98 \%$ of participants were of Dutch origin, which made subgroup analysis by ethnic groups not possible ${ }^{(12)}$. Moreover, most studies used selfor parent-reported questionnaires while it is well known that parents perceive children's HRQOL significantly different compared with children themselves ${ }^{(13,14)}$.

In the current study, we aimed to measure HRQOL in a multi-ethnic mix of 8-18-year-old obese children in Amsterdam, the Netherlands, in order to establish possible parent-proxy and ethnic-related differences. We hypothesized that: (i) obese children have a reduced HRQOL, as measured with the KIDSCREEN questionnaire, compared with an age- and sex-matched reference population; (ii) children with increasing BMI, waist circumference and fat percentage have a lower HRQOL; (iii) parents report lower HRQOL scores than self-reported HRQOL; and finally (iv) obese children of Moroccan, Turkish and Surinamese descent have poorer HRQOL than obese Caucasian children.

\section{Methods}

The study was conducted according to the guidelines of the Declaration of Helsinki and approved by the Ethics Review Board of the Slotervaart Hospital, Amsterdam.

\section{Study population}

All overweight and obese children (BMI $Z$-score $>2 \cdot 1$, aged 8-18 years) who were referred to the paediatric obesity out-patient clinic at the Slotervaart Hospital in 2012-2013 and their parents/guardians were administered two separate HRQOL questionnaires (KIDSCREEN-52 selfreport for children and a proxy questionnaire for their parent(s)/guardian(s) $\left.{ }^{(15)}\right)$ during an oral glucose tolerance test (as part of a diagnostic treatment protocol for childhood obesity). Subjects were excluded if they did not have sufficient understanding of the Dutch language or if children had a genetic syndrome or developmental disabilities. Part of this cohort is described in a previous paper, showing a heterogeneous group with no acute complications and some co-morbidities including metabolic syndrome (25\%), hypertension (20\%), impaired glucose metabolism (16\%) and hypercholesterolaemia $(13 \%)^{(4)}$.

\section{KIDSCREEN}

The paediatric HRQOL inventory (KIDSCREEN) was selected to measure HRQOL and was completed by the children and, if present, their proxy.

This questionnaire, with child and parent-proxy versions, has been validated for QOL assessment in children aged $8-18$ years $^{(15)}$. The fifty-two-item KIDSCREEN encompasses ten scales: (i) Physical Wellbeing (five items); (ii) Psychological Wellbeing (six items); (iii) Moods \& Emotions (seven items); (iv) Self-Perception (five items); (v) Autonomy (five items); (vi) Parent Relations \& Home Life (six items); (vii) Social Support \& Peers (six items); (viii) School Environment (six items); (ix) Bullying (three items); and (x) Financial Resources (three items) ${ }^{(12)}$. A 5-point Likert-type scale is used to assess either the frequency ('never'/'seldom'/'quite often'/'very often'/ 'always') or the intensity ('not at all'/'slightly'/'moderately'/ 'very'/'extremely') of certain behaviours, feelings or attitudes based on the recall period of 1 week $^{(15)}$. In the KIDSCREEN-10, ten items from the KIDSCREEN-52 are derived that measure global HRQOL; these are displayed in Table 1. Rasch scores were computed for each dimension and were transformed into $T$ values with a mean of 50 and an SD of 10, whereby higher scores indicate better HRQOL and well-being ${ }^{(16)}$. Published paediatric HRQOL inventory Dutch reference data of the KIDSCREEN-52 and KIDSCREEN-10 questionnaire were used for sex- and agespecific (8-11 years and 12-18 years) comparisons ${ }^{(16)}$. This reference group contains data of 1595 Dutch children aged $8-18$ years from different areas in the Netherlands ${ }^{(16)}$. The distribution of weight groups is comparable to the general Dutch population with 1407 (88.2\%) normal-weight children, $159(10.0 \%)$ overweight and twenty-nine $(1 \cdot 8 \%)$ obese children ${ }^{(2)}$.

\section{Physical examination}

Height was measured with a standardized stadiometer. Weight and body composition were measured in the fasting state, wearing light clothing, standing barefoot on

Table 1 Items of the KIDSCREEN-10†

1. Have you felt fit and well?

2. Have you felt full of energy?

3. Have you felt sad?

4. Have you felt lonely?

5. Have you had enough time for yourself?

6. Have you been able to do the things that you want to do in your free time?

7. Have your parent(s) treated you fairly?

8. Have you had fun with your friends?

9. Have you got on well at school?

10. Have you been able to pay attention?

†The ten self-reported items of the KIDSCREEN-52 that measure global health-related quality of life. 
metal foot-plates while holding the handles of the bioimpedance analyser (model BC-418; Tanita Europe, Amsterdam, The Netherlands), which measures weight to the nearest $0.1 \mathrm{~kg}$ and calculates estimates of fat percentage and fat (free) mass ${ }^{(17)}$. BMI and waist circumference were standardized according to sex and age using $Z$-scores, the SD score according to Dutch reference values ${ }^{(18,19)}$. Pubertal stage was assessed according to the Tanner staging ${ }^{(20)}$.

\section{Definitions}

Ethnicity was defined as Caucasian if both parents were Caucasian, and Moroccan or Turkish in the case that both parents were from that specific country. Ethnicity was defined as African Surinamese when both parents were from Suriname and originally of African descent. All other ethnicities or children with mixed ethnicities were collected as 'mixed'. Family structure was derived from the proxy KIDSCREEN-52 questionnaire where marital status and home situation were reported.

\section{Statistical analysis}

Substitution of missing values and calculation of the subscale scores were performed according to the KIDSCREEN manual $^{(16)}$. In the KIDSCREEN self-reports, 221 values were missing, a percentage of $1.4 \%$. In the parent-proxy reports 720 values were missing, a percentage of $6 \cdot 0 \%$. Differences between the reference group and the study group were tested with independent $t$ tests. Paired-sample $t$ tests were conducted to compare self- and parent-proxy reported HRQOL. Pearson correlations were used to explore the relationships between BMI $Z$-score and HRQOL subscales. Effect sizes, $d$, were calculated employing the estimated marginal means $\left(\right.$ mean $_{\text {sample }}-$ mean $\left._{\text {reference }} / \mathrm{SD}_{\text {pooled }}\right)$ and were interpreted as small $(0 \cdot 20-0 \cdot 50)$, moderate $(0 \cdot 51-0 \cdot 80)$ or large $(>0 \cdot 80)^{(21)}$. Variables with a skewed distribution were log-transformed before analysis.

Stepwise multiple regression analysis was used to test associations between the five self-reported subscales most often impaired in obese children (dependent variable) with respectively BMI $Z$-score, waist circumference $Z$ score and fat percentage (independent variables), with adjustment for the confounding variables of sex, age and family structure. A $P$ value of $<0.05$ was considered significant. We did not correct for multiple testing because of the exploratory nature of the study. Effect modification was tested by including product terms into the regression models, where $P<0 \cdot 1$ was considered significant. Statistical analyses were conducted using the statistical software package IBM SPSS Statistics version 21·0.

\section{Results}

\section{Sample characteristics}

In total, eleven children were excluded; nine did not have sufficient understanding of the Dutch language, one child was diagnosed with a genetic syndrome and one child had a developmental disability. Therefore, inclusion criteria were met by 308 of the 319 children and adolescents evaluated in the obesity out-patient clinic. Table 2 shows the baseline characteristics. The mean BMI was $30 \cdot 1$ (sD $5 \cdot 3$ ) $\mathrm{kg} / \mathrm{m}^{2}$, which corresponded to a mean BMI

Table 2 Baseline characteristics stratified by sex among multi-ethnic overweight and obese children aged 8-18 years from an obesity out-patient clinic, Amsterdam, the Netherlands, 2012-2013

\begin{tabular}{|c|c|c|c|c|c|c|c|}
\hline & \multicolumn{2}{|c|}{ Total (n 308) } & \multicolumn{2}{|c|}{ Boys ( $n 121 ; 39.4 \%$ ) } & \multicolumn{2}{|c|}{ Girls (n 187; 60.6\%) } & \multirow[b]{2}{*}{$P$ value } \\
\hline & Mean & SD & Mean & SD & Mean & SD & \\
\hline Age (years) & 12.5 & 2.56 & 12.5 & $2 \cdot 40$ & $12 \cdot 6$ & 2.66 & 0.792 \\
\hline BMl $\left(\mathrm{kg} / \mathrm{m}^{2}\right)$ & $30 \cdot 1$ & $5 \cdot 30$ & $30 \cdot 0$ & $5 \cdot 27$ & $30 \cdot 1$ & $5 \cdot 29$ & 0.955 \\
\hline BMI Z-score & $3 \cdot 16$ & 0.60 & $3 \cdot 30$ & 0.57 & 3.08 & 0.60 & 0.001 \\
\hline Waist circumference $(\mathrm{cm})$ & 97.8 & $14 \cdot 3$ & 99.9 & 14.5 & $96 \cdot 3$ & 14.0 & 0.037 \\
\hline Waist circumference Z-score & 3.85 & $1 \cdot 32$ & 4.41 & 1.53 & 3.43 & 0.94 & $<0.001$ \\
\hline Fat percentage & $37 \cdot 5$ & $6 \cdot 16$ & $35 \cdot 7$ & $6 \cdot 34$ & $39 \cdot 1$ & $5 \cdot 55$ & 0.007 \\
\hline \multirow[t]{2}{*}{ Global HRQOL score } & 44.9 & $5 \cdot 04$ & $44 \cdot 8$ & 4.94 & 44.9 & $5 \cdot 12$ & 0.869 \\
\hline & $n$ & $\%$ & $n$ & $\%$ & $n$ & $\%$ & \\
\hline Ethnicity & & & & & & & 0.248 \\
\hline Caucasian & 40 & 14.9 & 20 & 18.5 & 20 & $12 \cdot 4$ & \\
\hline Moroccan & 73 & $27 \cdot 1$ & 22 & $20 \cdot 4$ & 51 & 31.7 & \\
\hline Turkish & 82 & 30.9 & 38 & $36 \cdot 1$ & 44 & $27 \cdot 3$ & \\
\hline Surinamese & 37 & $13 \cdot 8$ & 14 & $13 \cdot 0$ & 23 & $14 \cdot 3$ & \\
\hline African & 22 & $7 \cdot 14$ & 5 & $4 \cdot 13$ & 17 & 9.09 & \\
\hline Mixed & 57 & $18 \cdot 5$ & 21 & $17 \cdot 4$ & 36 & $19 \cdot 3$ & \\
\hline BMI category† & & & & & & & $<0.001$ \\
\hline Overweight & 23 & $7 \cdot 47$ & 7 & $5 \cdot 79$ & 16 & 8.56 & \\
\hline Obesity & 98 & 31.6 & 25 & 20.5 & 73 & $38 \cdot 8$ & \\
\hline Morbidly obese & 187 & 61.0 & 89 & 73.8 & 98 & 52.7 & \\
\hline
\end{tabular}

HRQOL, health-related quality of life. 
$Z$-score of 3.2 (SD 0.6). Mean age was 12.5 (SD 2.56) years. There were no sex-specific differences with respect to baseline data, except that girls had a higher fat percentage, while boys had higher BMI and waist circumference $Z$-scores (Table 2). BMI $Z$-score was significantly lower in Caucasian children compared with ethnic children (mean $3 \cdot 0$ (sD 0.6) $v \cdot 3 \cdot 2$ (SD 0.6), $P=0 \cdot 045$ ). There were no differences between primary-school children and highschool children with respect to BMI $Z$-score, waist circumference $Z$-score, fat percentage and ethnicity (data not shown). In total, 213 parent(s)/guardian(s) of the included children completed the proxy questionnaire. The children represented by a parent-proxy report were younger than the children without parent-proxy reports (mean age 12.2 (SD 2.4) v. 13.2 (SD 2.8) years, $P=0.005$ ). There were no differences in mean BMI $Z$-score, waist circumference $Z$-score, fat percentage, HRQOL, sex, ethnicity or school between children with and without parent-proxy reports.

\section{Health-related quality of life}

\section{Self-report}

Overweight and obese children reported significantly lower HRQOL in the subscales of Physical Wellbeing and Self-Perception compared with age- and sex-matched Dutch reference values (Tables 3 and 4).

Children aged 8-11 years showed impairments in the HRQOL subscales Moods \& Emotions and Autonomy. Girls in this age group were bullied more often than the reference group, while this was not evident in boys. The high-school children (aged 12-18 years) reported higher scores in School Environment compared with the reference group. The highest reductions in all groups were observed in the subscales Physical Wellbeing and Self-Perception.

\section{Parent-proxy report}

Of the 213 parents, 150 were mothers (70.4\%), forty-seven were fathers $(22 \cdot 1 \%)$ and sixteen were others such as an aunt or grandparent (7.5\%). Parent(s)/guardian(s) reported significantly lower HRQOL scores for their overweight and obese children in the HRQOL subscales Physical Wellbeing (45.9 v. 54.0, $P<0.001)$ and Self-Perception (45.3 v. 51.7, $P<0.001$ ) compared with age- and sexmatched Dutch proxy reference values (data not shown).

For children aged 8-11 years, the parent-proxy questionnaires showed impairments in the HRQOL subscales Autonomy (50.6 (sD 8.3) v. 55.2 (SD 7.5), $P=0.001)$ and Social Support $(50 \cdot 8(\mathrm{sD} 10 \cdot 3) v .54 \cdot 3(\mathrm{SD} 8 \cdot 2), P=0 \cdot 018)$ and for 8-11-year-old girls lower scores for Moods \& Emotions (45.1 (sD 11.5) v. 49.3 (sD 9.4), $P=0.009$ ), compared with the reference group.

Parents of high-school children (aged 12-18 years) reported that their children were bullied more often than the reference group $(44 \cdot 1(\mathrm{SD} 12 \cdot 5)$ v. $47 \cdot 8(\mathrm{SD}=10 \cdot 9)$, $P=0.030)$ and reported higher scores on the subscales School Environment (54.6 (SD 11.4) v. 50.9 (SD 9.2),

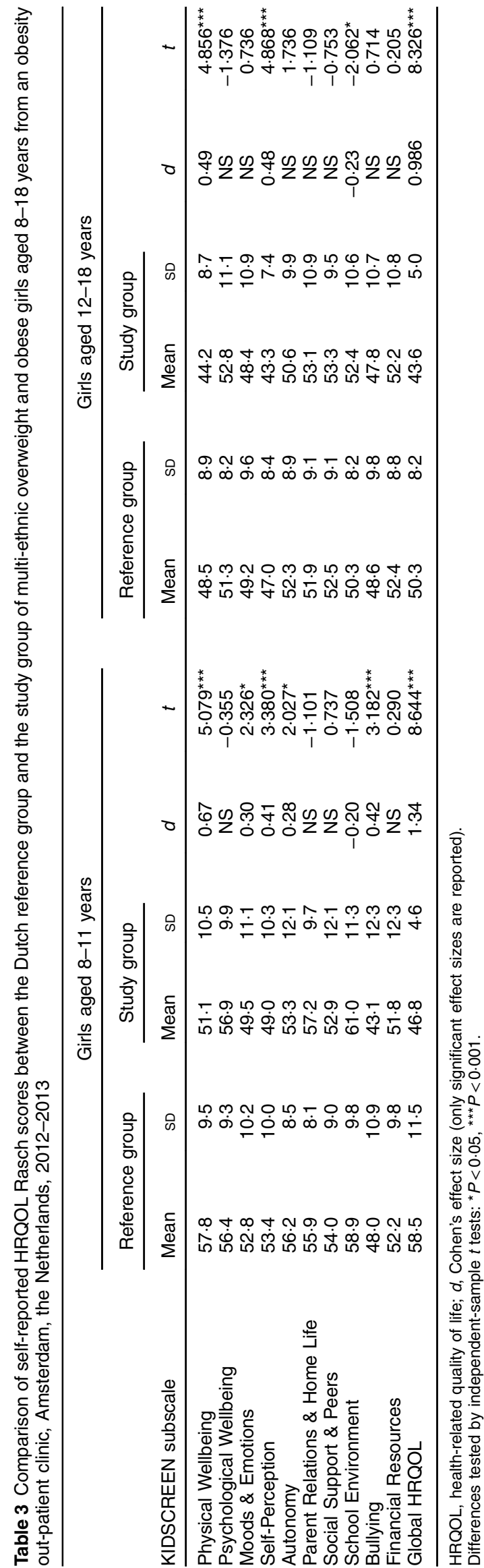


$P=0.025)$ and Psychological Wellbeing for boys $(55.5$ (SD 11.5) v. 52.2 (SD 9.7), $P=0.046$ ), compared with the reference group. The parent(s)/guardian(s) of 12-18-yearold girls reported reductions in Autonomy score compared with the reference group (50.8 (SD 10.6) v. 53.5 (SD 8.3), $P=0.046)$. There was no difference in parent-proxy scores for any of the subscales between ethnic groups.

\section{Comparisons and correlations of self-and parent-} proxy reported health-related quality of life

Figure 1 shows the differences between the self- and parent-proxy reported HRQOL for the ten subscales and global HRQOL. On the subscales Physical Wellbeing, Moods \& Emotions and Bullying, parents reported significantly worse HRQOL compared with self-report (Fig. 1).

There were significant correlations between self- and parent-proxy reported scores on the subscales Physical Wellbeing $(r=0 \cdot 191)$, Self-Perception $(r=0 \cdot 149)$, Parent Relations \& Home Life $(r=0.207)$, Social Support $(r=0 \cdot 306)$, School Environment $(r=0 \cdot 204)$ and Bullying $(r=0.339)$.

\section{Correlations of health-related quality of life and measures of obesity}

In univariate analyses, the subscale Physical Wellbeing was significantly correlated with BMI $Z$-score $(r=-0 \cdot 123$, $P=0.033)$ and fat percentage $(r=-0.303, P=0.030)$. The subscale Self-Perception showed significant correlations with BMI $Z$-score $(r=-0 \cdot 123, P=0.033)$ and fat percentage $(r=-0.354, P=0.001)$. Autonomy was correlated with fat percentage $(r=-0.252, P=0.014)$. There were no correlations between ethnic groups and any of the HRQOL subscales.

\section{Regression analyses}

In linear regression analyses, a significant interaction term of BMI $Z$-score and ethnicity was found. Therefore, regression analyses were performed separately for the ethnic minorities and Caucasian children. No significant associations were found in the ethnic minorities group; however, in Caucasian children there were significant associations for respectively lower scores on the subscales Moods \& Emotions $(\beta=-3 \cdot 982,95 \%$ CI $-6 \cdot 846,-1 \cdot 118$, $P=0 \cdot 009)$, Self-Perception $(\beta=-2 \cdot 698$, $95 \%$ CI $-4 \cdot 884$, $-0.512, \quad P=0.018)$ and Bullying $(\beta=-3.757,95 \% \mathrm{CI}$ $-6.668,-0.846, P=0.014)$ with higher BMI $Z$-score, all after adjustments for age, sex and family structure.

Lower scores on the subscale Physical Wellbeing were significantly associated with higher waist circumference $(\beta=-0.023,95 \%$ CI $-0.41,-0.05, P=0.014)$ in multivariate analysis with adjustments for age, sex and family structure. In multivariate analyses, Self-Perception was associated with age and sex. School Environment was 


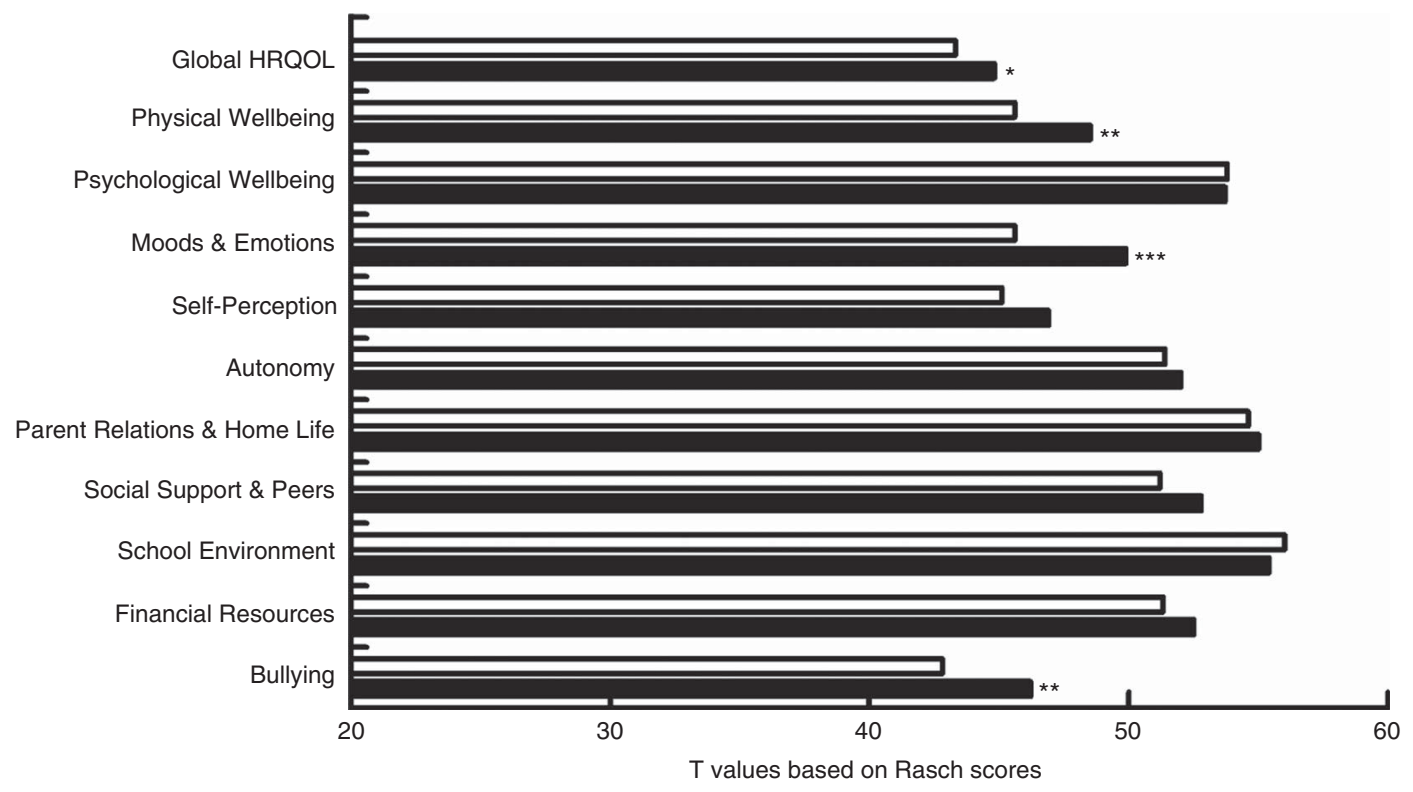

Fig. 1 Self-reported ( $\square$ ) and parent-proxy reported ( $\square$ ) health-related quality of life (HRQOL) scores, global and across the subscales of the KIDSCREEN questionnaire, among overweight and obese children aged 8-18 years and their parents from an obesity out-patient clinic, Amsterdam, the Netherlands, 2012-2013. Differences tested by paired-samples $t$ tests: ${ }^{*} P<0.05$, ${ }^{* *} P<0.01,{ }^{* *} P<0.001$

associated only with age, not with sex or family structure (data not shown).

In multivariate analyses, the association of Physical Wellbeing with fat percentage was lost $(\beta=-0 \cdot 076$, 95\% CI $-0 \cdot 163,0 \cdot 010, P=0 \cdot 081)$ after adjustment for age, sex and family structure. There was a significant negative association of Self-Perception with fat percentage $(\beta=-0.054,95 \%$ CI $-0.106,0.002, P=0.041)$ and of Autonomy with fat percentage $(\beta=-0.091,95 \% \mathrm{CI}-0.173,0.009, P=0.030)$.

\section{Discussion}

The present study is the first to determine HRQOL in obese Dutch children across different ethnic subgroups. We found that treatment-seeking obese multi-ethnic children aged 8-18 years experience significantly worse HRQOL, and specifically in the subscales Physical Wellbeing and Self-Perception, compared with the Dutch age- and sex-matched reference population. These findings are supported by previous studies in overweight and obese children ${ }^{(9,10,22,23)}$ including one study in eighty Dutch severely obese children (BMI $Z$-score $\geq 3.0$ or BMI $Z$-score $\geq 2 \cdot 3$ with co-morbidity) using the EuroQol selfreported questionnaires ${ }^{(22)}$. Surprisingly, we did not find a relationship between the severity of obesity measured by increasing BMI $Z$-score, waist circumference $Z$-score and fat percentage and global HRQOL. However, the subscale Physical Wellbeing was negatively correlated with BMI $Z$-score, waist circumference $Z$-score and fat percentage. Several other studies have found a significant relationship between waist circumference and impaired QOL in both adults $^{(24)}$ and children ${ }^{(25,26)}$. It is well known that overweight and obese children experience less physical well-being compared with lean children due to obesityrelated complaints such as shortness of breath during exercise, joint pain and a low physical fitness level ${ }^{(27,28)}$. Low self-perception could be caused by the stigma of childhood obesity and the concomitant dissatisfaction with their appearance ${ }^{(29)}$. Indeed, children in this cohort reported the constant desire to change their body and envied other children's physique, and children with a higher fat percentage reported significantly lower scores on the subscale Self-Perception, which could not be attributed to confounding factors such as age, sex and family structure.

Sociodemographic factors may be important in QOL. However, we did not find significant disparities in subscales of HRQOL between Caucasian children and obese children of Turkish, Moroccan, Surinamese or African descent in either the self- or parent-proxy reported scores. This is comparable with Wallander et al., who found no differences in QOL in 4824 Caucasian, Afro-American and Hispanic school children (mean age 11.2 years, $46 \%$ overweight or obese) ${ }^{(11)}$. In contrast, several other studies did find significant differences in QOL among ethnic groups $^{(9,12,30)}$. Trevino et al. showed that QOL in 4979 sixth graders in the USA was better for Afro-American than for Caucasian children, even after adjustment for BMI $Z$-score ${ }^{(30)}$. This is in concordance with a Dutch study that found higher scores on several HRQOL subscales in fiftyfive non-Dutch children compared with 3172 children of Dutch origin aged 5-6 years ${ }^{(12)}$ and Fallon et al. who reported that being overweight has more impact on QOL in white compared with black adolescents ${ }^{(9)}$. We found an 
ethnic-specific negative correlation between the HRQOL subscales Moods \& Emotions, Self-Perception and Bullying and BMI $Z$-score, even after adjustment for confounding factors such as age, sex and family situation. These correlations were present in Caucasian children, although not in the other ethnic groups, therefore suggesting a different pattern of the relationship between HRQOL and BMI $Z$-score in Caucasian children. Perhaps the severity of obesity has more impact on Caucasian children due to different cultural interpretations of (over)eating and (childhood) obesity ${ }^{(31)}$.

In confirmation of our hypothesis and other stu$\operatorname{dies}^{(13,14,32)}$, parents reported their obese children to have worse HRQOL than indicated by the self-reported scores of the obese children on the global HRQOL scale and the subscales Physical Wellbeing, Moods \& Emotions and Bullying. It is not entirely clear why parents report lower HRQOL scores than their children. One of the hypotheses is that parents struggling with overweight or obesity themselves assign their own stress and difficulties to the QOL of their children ${ }^{(32)}$. In a broader sense, Steinsbekk et al. indeed showed that the parent-reported QOL of obese children was attributable to the high levels of psychopathology of the parents themselves ${ }^{(14)}$. However, since parents have such a big influence on children's behaviour, we believe intervention programmes aiming to improve HRQOL should be directed to both parents and children.

The literature shows that obese school-aged children are more likely to be a victim of bullying behaviour than their normal-weight peers ${ }^{(33)}$. Indeed, we did find significantly lower bullying scores in obese girls aged 8-11 years compared with their age- and sex-matched reference. This is troubling, because bullying too often leads to violence, low self-esteem, depression and even suicide ${ }^{(33)}$. Therefore, school-based anti-bullying intervention counsellors should be aware of high-risk groups such as these girls because they are in need of a safe environment. Surprisingly, both self- and parent-proxy report showed a higher score on the subscale of School Environment for overweight and obese high-school children. The questions in this subscale include 'Have you enjoyed going to school?' and 'Have you got along well with your teachers?' This high score could be caused by less social contact with peers, as we did find significantly more bullying in girls aged $8-11$ years compared with the reference group and a significant association between BMI $Z$-score and bullying in Caucasian children. Therefore, we hypothesize that these children might spend more time studying and build a better relationship with their teachers.

The strength of the present study is the determination of HRQOL in a large sample of multi-ethnic children in Northern Europe. Unlike previous studies, our study measured children's weight and height directly and employed standard HRQOL scales. However, we have to acknowledge several limitations. The observational design prevents us from examining causal effects of BMI $Z$-score
On HRQOL. Selection bias may have occurred since participants were referred to a paediatric obesity out-patient clinic in Amsterdam. Our findings cannot be generalized to the larger group of obese children seen or treated by general health practitioners in smaller cities in the Netherlands. In Amsterdam, 34.9\% of the population is of non-Western descent; this is in contrast to other regions of the Netherlands where generally $12 \%$ of the population is of non-Western descent ${ }^{(3)}$. In our study sample the majority of the non-Western children were of Turkish or Moroccan descent (58\%) and only $15 \%$ of the total cohort was Caucasian. Moreover, we did not have data to determine socio-economic status. Also, other factors could have influenced QOL such as conflicts in the family, mental or physical illnesses of parents or financial problems. It is recommended that epidemiological and longitudinal trials regarding obesity and its treatment add HRQOL as a critical end point. Since the correlations of the KIDSCREEN-52 were relatively low, an obesity-specific HRQOL questionnaire is preferable since this has a higher specificity for obesity-related QOL.

To conclude, treatment-seeking obese children had significantly worse HRQOL, specifically physical wellbeing and self-perception, compared with the Dutch ageand sex-matched reference population in both self- and parent-proxy reported HRQOL questionnaires. We believe intervention programmes aiming to improve HRQOL should be directed to both parents and children, while ethnic-specific programmes to enhance HRQOL seem of less importance.

\section{Acknowledgements}

Financial support: This research received no specific grant from any funding agency in the public, commercial or notfor-profit sectors. No honorarium, grant or other payment was given to anyone to conduct the research or produce the manuscript. Conflict of interest: The authors declare no conflict of interest. Authorship: N.N.E.R. wrote the first draft of the manuscript and was responsible for the data collection. N.N.E.R., M.v.V. and M.D. contributed in concept and design, analysis and interpretation of data and revision. M.d.W. contributed in analysis and interpretation of data, drafting of the article and revision. I.A.v.R., J.B. and D.B. contributed to the design, data collection, interpretation of data and revision. All authors have approved the final version of the article for publication. Ethics of buman subject participation: The study was conducted according to the guidelines of the Declaration of Helsinki and approved by the Ethics Review Board of the Slotervaart Hospital, Amsterdam.

\section{References}

1. James PT, Leach R, Kalamara E et al. (2001) The worldwide obesity epidemic. Obes Res 9 , Suppl. 4, 228S-233S. 
2. Voorrips LE \& van Hilten O (2012) Gezondheid en zorg in cijfers. Den Haag: Centraal Bureau voor de Statistiek.

3. Centraal Bureau voor de Statistiek (2013) Bevolking: leeftijd, herkomstgroepering, geslacht en regio. Den Haag: Centraal Bureau voor de Statistiek.

4. Van Vliet M, Von Rosenstiel IA, Schindhelm RK et al. (2009) Ethnic differences in cardiometabolic risk profile in an overweight/obese paediatric cohort in the Netherlands: a cross-sectional study. Cardiovasc Diabetol 8, 2.

5. Daniels SR (2009) Complications of obesity in children and adolescents. Int J Obes (Lond) 33, Suppl. 1, S60-S65.

6. Pedersen A, Zachariae R \& Bovbjerg DH (2010) Influence of psychological stress on upper respiratory infection; a metaanalysis of prospective studies. Psychosom Med 72, 823-832.

7. de Beer MH, Hofsteenge GH, Koot HM et al. (2007) Healthrelated-quality-of-life in obese adolescents is decreased and inversely related to BMI. Acta Paediatr 96, 710-714.

8. Wille N, Bullinger M, Holl R et al. (2010) Research HealthRelated quality of life in overweight and obese youths: results of a multicenter study. Health Qual Life Outcomes 8, 36-44.

9. Fallon EM, Tanofsky-Kraff M, Norman AC et al. (2005) Health-related quality of life in overweight and nonoverweight black and white adolescents. J Pediatr 147, 443-450.

10. Schwimmer JB, Burwinkle TM \& Varni JW (2003) Healthrelated quality of life of severely obese children and adolescents. JAMA 289, 1813-1819.

11. Wallander J, Kerbawy S, Toomey S et al. (2013) Is obesity associated with reduced health-related quality of life in Latino, black and white children in the community? Int $J$ Obes (Lond) 37, 920-925.

12. van Grieken A, Veldhuis L, Renders CM et al. (2013) Impaired parent-reported health-related quality of life of underweight and obese children at elementary school entry. Qual Life Res 22, 1-12.

13. Bianchini JA, da Silva DF, Nardo CC et al. (2013) Parentproxy perception of overweight adolescents' health-related quality of life is different according to adolescent gender and age and parent gender. Eur J Pediatr 172, 1371-1377.

14. Steinsbekk S, Jozefiak T, Odegard R et al. (2009) Impaired parent-reported quality of life in treatment-seeking children with obesity is mediated by high levels of psychopathology. Qual Life Res 18, 1159-1167.

15. Ravens-Sieberer U, Gosch A, Rajmil L et al. (2005) KIDSCREEN-52 quality-of-life measure for children and adolescents. Expert Rev Pharmacoecon Outcomes Res 5 , 353-364.

16. The Kidscreen Group Europe (2006) The Kidscreen Questionnaires - Quality of Life Questionnaires for Children and Adolescents. Handbook. Lengerich: Pabst Science Publishers.

17. Pietrobelli A, Rubiano F, St-Onge $M$ et al. (2004) New bioimpedance analysis system: improved phenotyping with whole-body analysis. Eur J Clin Nutr 58, 1479-1484.
18. Fredriks AM, van Buuren S, Fekkes M et al. (2005) Are age references for waist circumference, hip circumference and waist-hip ratio in Dutch children useful in clinical practice? Eur J Pediatr 164, 216-222.

19. Fredriks AM, Van Buuren S, Wit JM et al. (2000) Body index measurements in 1996-7, compared with 1980. Arch Dis Child 82, 107-112.

20. Tanner T (1962) Growth at Adolescence: With a General Consideration of the Effects of Hereditary and Environmental Factors Upon Growth and Maturation from Birth to Maturity, 2nd ed. Oxford: Blackwell Scientific.

21. Cohen J (1988) Statistical Power Analysis for the Behavioral Sciences. Hillsdale, NJ: Lawrence Erlbaum.

22. Makkes S, Renders CM, Bosmans JE et al. (2013) Cardiometabolic risk factors and quality of life in severely obese children and adolescents in the Netherlands. BMC Pediatr 13, 62 .

23. Wu XY, Ohinmaa A \& Veugelers PJ (2012) Diet quality, physical activity, body weight and health-related quality of life among grade 5 students in Canada. Public Health Nutr 15, 75-81.

24. Lean M, Han T \& Seidell J (1998) Impairment of health and quality of life in people with large waist circumference. Lancet 351, 853-856.

25. Gopinath B, Baur LA, Burlutsky G et al. (2013) Adiposity adversely influences quality of life among adolescents. J Adolesc Health 52, 649-653.

26. Kesztyus D, Wirt T, Kobel S et al. (2013) Is central obesity associated with poorer health and health-related quality of life in primary school children? Cross-sectional results from the Baden-Württemberg Study. BMC Public Health 13, 260.

27. Shultz S, Anner J \& Hills AP (2009) Paediatric obesity, physical activity and the musculoskeletal system. Obes Rev 10, 576-582.

28. Parameswaran K, Todd DC \& Soth M (2006) Altered respiratory physiology in obesity. Can Respir $J \mathbf{1 3}$, 203-210.

29. Mustillo SA, Hendrix KL \& Schafer MH (2012) Trajectories of body mass and self-concept in black and white girls the lingering effects of stigma. J Health Soc Behav 53, 2-16.

30. Trevino R, Pham $\mathrm{T}$ \& Edelstein S (2013) Obesity and preference-weighted quality of life of ethnically diverse middle school children: the HEALTHY study. J Obes $\mathbf{2 0 1 3}$, 206074.

31. Nicolaou M, Doak CM, van Dam RM et al. (2009) Cultural and social influences on food consumption in Dutch residents of Turkish and Moroccan origin: a qualitative study. J Nutr Educ Behav 41, 232-241.

32. Guilfoyle SM, Zeller MH \& Modi AC (2010) Parenting stress impacts obesity-specific health-related quality of life in a pediatric obesity treatment-seeking sample. J Dev Behav Pediatr 31, 17-25.

33. Lemstra ME, Nielsen G, Rogers MR et al. (2012) Risk indicators and outcomes associated with bullying in youth aged 9-15 years. Can J Public Health 103, 9-13. 